\title{
NONEXISTENCE OF LINEAR OPERATORS EXTENDING LIPSCHITZ (PSEUDO)METRIC
}

\author{
DUŠAN REPOVŠ (LJUBLJANA) AND MICHAEL ZARICHNYI (LVIV AND RZESZÓW)
}

\begin{abstract}
We present an example of a zero-dimensional compact metric space $X$ and its closed subspace $A$ such that there is no continuous linear extension operator for the Lipschitz pseudometrics on $A$ to the Lipschitz pseudometrics on $X$. The construction is based on results of A. Brudnyi and Yu. Brudnyi concerning linear extension operators for Lipschitz functions.
\end{abstract}

\section{INTRODUCTION}

The problem of extensions of metric has a long history. Hausdorff was the first to prove that any continuous metric defined on a closed subset of a metrizable space can be extended to a continuous metric defined on the whole space. Bessaga [5, 6] considered the problem of existence of linear extension operators for metrics and provided a partial solution of this problem. The problem was completely solved by Banakh [3] (see also a short proof in [17]).

The problem of extending Lipschitz and uniform (pseudo)metrics was considered in [11. It is well-known that any Lipschitz pseudometric defined on a closed subset of a metric space admits an extension which is a Lipschitz pseudometric defined on the whole space. Recently, an extension operator for Lipschitz and uniform pseudometrics was constructed in [4 which preserves the Lipschitz norm and has many nice properties.

In the present paper we shall consider the problem of existence of linear extension operators for Lipschitz pseudometric. To the best of our knowledge, no affirmative results are known in this direction. Our goal is to construct a counterexample, i.e. show that there exists a subset of a zero-dimensional compact metric space for which there is no such extension operator (recall that a space is zero-dimensional if its topology possesses a base consisting of sets which are open and closed). The example is based on results from [7] concerning the linear extension operators for Lipschitz functions.

Note that conditions for existence of extensions of continuous functions are often equivalent to those for existence of extensions of continuous pseudometrics (cf. e.g. [1, 2, 12, 13]). It turns out that in the case of linear extension operators, one is able to procede at least in one direction, namely from pseudometrics to functions.

\section{Preliminaries}

Let $(X, \varrho)$ be a compact metric space. Given a subset $A$ of $X$, a pseudometric $d$ on $A$ is said to be Lipschitz if there exists $C>0$ such that

$$
d(x, y) \leq C \varrho(x, y) \text { for any } x, y \in A .
$$

Next, a map $f: X \rightarrow Y$, where $(Y, d)$ is a metric space, is called C-Lipschitz, $C>0$, if

$$
d(f(x), f(y)) \leq C \varrho(x, y) \text { for every } x, y \in X .
$$

1991 Mathematics Subject Classification. 26A16, 54C20, 54E35, 54E40.

Key words and phrases. Lipschitz pseudometric, extension operator, Lipschitz function, zero-dimensional compactum, bi-Lipschitz equivalence.

This research was supported by the Slovenian Research Agency grants P1-0292-0101 and J1-2057-0101. The authors are indebted to Ihor Stasyuk for pointing an error in the preliminary version. We also thank all referees for their remarks. 
If the knowledge of $C$ is not important, we shall use the term "Lipschitz map" instead of "C-Lipschitz map".

The set of reals $\mathbb{R}$ is endowed with the standard metric $|x-y|$. A bijective map $f$ of metric spaces is said to be bi-Lipschitz if both $f$ and $f^{-1}$ are Lipschitz. Metric spaces are called bi-Lipschitz equivalent if there exists some bi-Lipschitz map between them.

Until the end of this section, $A$ will denote a nonempty closed subset in a compact metric space $(X, \varrho)$. Denote by $\mathcal{P} \mathcal{M}_{\text {Lip }}(A)$ (resp. $\left.\mathcal{M}_{\text {Lip }}(A), \mathcal{C}_{\text {Lip }}(A)\right)$ the set of all Lipschitz pseudometrics (resp. metrics, functions) on $A$. The set $\mathcal{P} \mathcal{M}_{\text {Lip }}(A)$ (resp. $\mathcal{C}_{\text {Lip }}(A)$ ) is a cone (resp. linear space) with respect to the operations of pointwise addition and multiplication by scalar.

We endow $\mathcal{P} \mathcal{M}_{\text {Lip }}(A)$ with the norm $\|\cdot\|_{\mathcal{P} \mathcal{M}_{\text {Lip }}(A)}$ which is defined as follows:

$$
\|d\|_{\mathcal{P M}_{\text {Lip }}(A)}=\sup \left\{\frac{d(x, y)}{\varrho(x, y)} \mid x \neq y\right\}
$$

and we put on $\mathcal{C}_{\text {Lip }}(A)$ the seminorm $\|\cdot\|_{\mathcal{C}_{\text {Lip }}(A)}$ which is defined as follows:

$$
\|f\|_{\mathcal{C}_{\text {Lip }}(A)}=\sup \left\{\frac{|f(x)-f(y)|}{\varrho(x, y)} \mid x \neq y\right\}
$$

(in the sequel, we shall abbreviate $\|\cdot\|_{\mathcal{P} \mathcal{M}_{\text {Lip }}(A)}$ and $\|\cdot\|_{\mathcal{C}_{\text {Lip }}(A)}$ by $\left.\|\cdot\|_{A}\right)$.

We say that a map $u: \mathcal{P} \mathcal{M}_{\text {Lip }}(A) \rightarrow \mathcal{P} \mathcal{M}_{\text {Lip }}(X)$ is an extension operator for Lipschitz pseudometrics if the following holds:

(1) $u$ is linear, i.e.

$$
\left.u\left(d_{1}+d_{2}\right)=u\left(d_{1}\right)+u\left(d_{2}\right), u(\lambda d)=\lambda u(d) \text { for every } d_{1}, d_{2} \in \mathcal{P} \mathcal{M}_{\mathrm{Lip}}(A), \lambda \in \mathbb{R}_{+}\right) ;
$$

(2) $u(d) \mid(A \times A)=d$, for every $d \in \mathcal{P} \mathcal{M}_{\text {Lip }}(A)$; and

(3) $u$ is continuous in the sense that

$$
\|u\|=\sup \left\{\|u(d)\|_{X} \mid\|d\|_{A} \leq 1\right\} \text { is finite. }
$$

This definition is a natural analogue of that introduced in 7 for the extensions of Lipschitz functions. The following notation was introduced in [7:

$$
\lambda(A, X)=\inf \left\{\|u\| \mid u \text { is a linear extension operator from } \mathcal{C}_{\text {Lip }}(A) \text { to } \mathcal{C}_{\text {Lip }}(X)\right\} .
$$

Similarly, we define

$$
\begin{aligned}
\Lambda(A, X)= & \inf \{\|u\| \mid u \text { is a linear extension operator } \\
& \left.\mathcal{P} \mathcal{M}_{\text {Lip }}(A) \rightarrow \mathcal{P} \mathcal{M}_{\text {Lip }}(X)\right\} .
\end{aligned}
$$

It can be easily proved that

$$
\Lambda(X)=\sup \{\Lambda(A, X) \mid A \subset X\}
$$

is a bi-Lipschitz invariant of a metric space $X$, i.e.

$$
\Lambda(Y)=\Lambda\left(Y^{\prime}\right) \text { whenever } Y \text { and } Y^{\prime} \text { are bi-Lipschitz equivalent. }
$$

\section{Auxiliary Results}

Given a metric space $X=(X, d)$ and $c>0$, we denote the metric space $(X, c d)$ by $c X$.

Lemma 3.1. For a metric space $(X, d), S \subset X$, and $c>0$, we have $\lambda(c S, c X)=\lambda(S, X)$.

Proof. Let $\varphi \in \mathcal{C}_{\text {Lip }}(c S)$ and $\|\varphi\|_{c S}=K$. Then $\varphi$ can also be considered as an element of $\mathcal{C}_{\text {Lip }}(S)$ with $\|\varphi\|_{S}=K / c$. There exists an extension $\bar{\varphi}: X \rightarrow \mathbb{R}$ of $\varphi$ with $\|\bar{\varphi}\|_{X} \leq(K \lambda(S, X)) / c$. Considering $\bar{\varphi}$ as an element of $\mathcal{C}_{\text {Lip }}(c X)$, we see that $\|\bar{\varphi}\|_{c X} \leq(K \lambda(S, X))$. Therefore $\lambda(c S, c X) \leq \lambda(S, X)$. Arguing similarly, one can prove also the opposite inequality.

Lemma 3.2. Let a metric pair $\left(S_{1}, X_{1}\right)$ be a retract of a metric pair $(S, X)$ under a 1-Lipschitz retraction. Then $\lambda\left(S_{1}, X_{1}\right) \leq \lambda(S, X)$. 
Proof. Let $r: X \rightarrow X_{1}$ be a 1-Lipschitz retraction such that $r(S)=S_{1}$. Given a Lipschitz function $f: S_{1} \rightarrow \mathbb{R}$, we see that $f \circ(r \mid S)$ is a Lipschitz function on $S$ with $\|f \circ(r \mid S)\|_{S}=\|f\|_{S_{1}}$. There is an extension $g: X \rightarrow \mathbb{R}$ of $f \circ(r \mid S)$ with $\|g\|_{X} \leq \lambda(S, X)\|f \circ(r \mid S)\|_{S}$. Then $g \mid X_{1}$ is an extension of $f$ over $X_{1}$ with $\left\|g \mid X_{1}\right\|_{X_{1}} \leq \lambda(S, X)\|f\|_{S_{1}}$.

Proposition 3.3. Let $S$ be a closed subset of a compact metric space $X$ with $|S| \geq 2$. Then the following are equivalent:

(1) there exists a continuous linear extension operator

$$
\mathcal{P} \mathcal{M}_{\text {Lip }}(S) \rightarrow \mathcal{P} \mathcal{M}_{\text {Lip }}(X)
$$

and

(2) there exists a continuous linear extension operator

$$
\mathcal{M}_{\text {Lip }}(S) \rightarrow \mathcal{M}_{\text {Lip }}(X)
$$

Proof. $(1) \Rightarrow(2)$. Let $u: \mathcal{P} \mathcal{M}_{\text {Lip }}(S) \rightarrow \mathcal{P} \mathcal{M}_{\text {Lip }}(X)$ be a continuous linear extension operator.

Note first that there exists $\tilde{\varrho} \in \mathcal{P} \mathcal{M}_{\text {Lip }}(X)$ such that $\tilde{\varrho}(x, y)=0$ if and only if $(x, y) \in$ $(S \times S) \cup \Delta_{X}$ (by $\Delta_{X}$ we denote the diagonal of $X$ ). In order to construct $\tilde{\varrho}$, consider for any $x, y \in X \backslash S$ with $x \neq y$, the pseudometric $\varrho_{x y}$ on $S \cup\{x, y\}$, defined by

$$
\varrho_{x y} \mid(S \times S)=0, \varrho_{x y}(x, y)=\varrho_{x y}(x, s)=\varrho_{x y}(y, s)=1
$$

for any $s \in S$. Denoting by $d$ the original metric on $X$ we see that

$$
\begin{aligned}
& \varrho_{x y}(x, y)=1=(1 / d(x, y)) d(x, y), \\
& \varrho_{x y}(x, s)=1 \leq(1 / d(x, S)) d(x, s), \\
& \varrho_{x y}(y, s)=1 \leq(1 / d(y, S)) d(y, s)
\end{aligned}
$$

for any $s \in S$. Therefore, $\varrho_{x y}$ is a Lipschitz pseudometric with the Lipschitz constant

$$
\max \{1 / d(x, y), 1 / d(x, S), 1 / d(y, S)\} .
$$

By a result of Luukkainen [11, Theorem 6.15], there exists a Lipschitz pseudometric $\tilde{\varrho}_{x y}$ on $X$ which is an extension of $\varrho_{x y}$.

There exist (necessarily disjoint) neighborhoods $U_{x y}$ and $V_{x y}$ of $x$ and $y$ respectively, such that $\tilde{\varrho}_{x y}\left(x^{\prime}, y^{\prime}\right) \neq 0$, for every $x^{\prime} \in U_{x y}$ and $y^{\prime} \in V_{x y}$. The family

$$
\left\{U_{x y} \times V_{x y} \mid x, y \in((X \backslash S) \times(X \backslash S)) \backslash \Delta_{X}\right\}
$$

forms an open cover of $((X \backslash S) \times(X \backslash S)) \backslash \Delta_{X}$ and, by separability of the latter set, there exists a sequence $\left(x_{i}, y_{i}\right)$ in $((X \backslash S) \times(X \backslash S)) \backslash \Delta_{X}$ such that

$$
\bigcup\left\{U_{x_{i} y_{i}} \times V_{x_{i} y_{i}} \mid i \in \mathbb{N}\right\}=((X \backslash S) \times(X \backslash S)) \backslash \Delta_{X} .
$$

Let

$$
\tilde{\varrho}=\sum_{i=1}^{\infty} \frac{\tilde{\varrho}_{x_{i} y_{i}}}{2^{i}\left\|\tilde{\varrho}_{x_{i} y_{i}}\right\|_{X}} .
$$

Then, obviously, $\tilde{\varrho} \in \mathcal{P} \mathcal{M}_{\mathrm{Lip}}(X)$ is as required.

Now define an operator $\tilde{u}: \mathcal{M}_{\text {Lip }}(S) \rightarrow \mathcal{M}_{\text {Lip }}(X)$ as follows. Let $x_{0}, y_{0} \in S, x_{0} \neq y_{0}$. Let $\tilde{u}(\delta)=u(\delta)+\delta\left(x_{0}, y_{0}\right) \tilde{\varrho}$, for any $\delta \in \mathcal{M}_{\text {Lip }}(S)$. We leave it to the reader to easily verify that $\tilde{u}$ is indeed a continuous extension operator.

$(2) \Rightarrow(1)$. We are going to show that $\mathcal{M}_{\text {Lip }}(S)$ is dense in $\mathcal{P} \mathcal{M}_{\text {Lip }}(S)$. Let $\varepsilon>0$. Given $\varrho \in \mathcal{P} \mathcal{M}_{\text {Lip }}(S)$, we see that $\varrho_{1}=\varrho+\varepsilon d^{\prime}$, where $d^{\prime}$ is the original metric on $S$, is an element of $\mathcal{M}_{\text {Lip }}(S)$ with $\left\|\varrho-\varrho_{1}\right\|_{S} \leq \varepsilon$.

Let $u: \mathcal{M}_{\text {Lip }}(S) \rightarrow \mathcal{M}_{\text {Lip }}(X)$ be a continuous linear extension operator. Since $\mathcal{M}_{\text {Lip }}(S)$ is dense in $\mathcal{P} \mathcal{M}_{\text {Lip }}(S)$ and the space $\mathcal{P} \mathcal{M}_{\text {Lip }}(X)$ is complete, there exists a unique continuous extension $\tilde{u}: \mathcal{P} \mathcal{M}_{\text {Lip }}(S) \rightarrow \mathcal{P} \mathcal{M}_{\text {Lip }}(X)$ of $u$. Obviously, $\tilde{u}$ is a continuous linear extension operator. 


\section{The Main Result}

The following is the main result of this paper.

Theorem 4.1. There exists a closed subspace A of a zero-dimensional compact metric space $X$ for which there is no extension operator for Lipschitz pseudometrics.

Proof. We first recall some results from $[7]$. Let $\mathbb{Z}_{1}^{n}(l)$ stand for $\mathbb{Z}^{n} \cap[-l, l]^{n}$ endowed with the $\ell_{1}$-metric. It was proved in [7, Lemma 10.5] that there exists $c_{1}>0$ satisfying the following condition: For any natural $n$ there exists an integer $l(n)>0$ and a subset $Y_{n} \subset \mathbb{Z}_{1}^{n}(l(n))$ such that

$$
\lambda\left(Y_{n}, \mathbb{Z}_{1}^{n}(l(n))\right) \geq c_{1} \sqrt{n}
$$

Let

$$
X=\left(\coprod_{n=1}^{\infty} \frac{1}{n l(n)} \mathbb{Z}_{1}^{n}(l(n))\right) / \sim,
$$

where $\sim$ is the equivalence relation which identifies all the origins, be the bouquet of the spaces $\mathbb{Z}_{1}^{n}(l(n)), n \in \mathbb{N}$. We naturally identify every

$$
\frac{1}{n l(n)} \mathbb{Z}_{1}^{n}(l(n)), n \in \mathbb{N},
$$

with its copy, which we denote by $X_{n}$, in $X$. The space $X$ is endowed with the maximal metric $\varrho$ inducing the original metric on $X_{n}$, for every $n \in \mathbb{N}$. Obviously, $X$ is a compact metric space. One can easily see that $X$ is zero-dimensional.

Let

$$
S_{n}=\frac{1}{n l(n)} Y_{n} \subset X_{n}, n \in \mathbb{N}
$$

Suppose that there exists an extension operator $u: \mathcal{P} \mathcal{M}_{\text {Lip }}(S) \rightarrow \mathcal{P} \mathcal{M}_{\text {Lip }}(X)$, where

$$
S=\left(\coprod_{n=1}^{\infty} S_{n}\right) / \sim \subset X .
$$

By $\mathcal{C}_{\text {Lip }}(X, 0)\left(\right.$ resp. $\left.\mathcal{C}_{\text {Lip }}(S, 0)\right)$ we denote the set of functions from $\mathcal{C}_{\text {Lip }}(X)\left(\right.$ resp. $\left.\mathcal{C}_{\text {Lip }}(S)\right)$ that vanish at $0 \in X$ and by $\mathcal{C}_{\text {Lip }}^{+}(X, 0)$ (resp. $\left.\mathcal{C}_{\text {Lip }}^{+}(S, 0)\right)$ we denote the set of nonnegative functions from $\mathcal{C}_{\text {Lip }}(X, 0)$ (resp. $\mathcal{C}_{\text {Lip }}(S, 0)$ ).

For any $x \in S \backslash\{0\}$, we denote by $f_{x}: S \rightarrow \mathbb{R}$ the function defined as follows:

$$
f_{x}(x)=1 \text { and } f_{x}(y)=0 \text {, whenever } y \neq x .
$$

Then $f_{x} \in \mathcal{C}_{\text {Lip }}^{+}(S, 0)$ and the collection $\left\{f_{x} \mid x \in S \backslash\{0\}\right\}$ forms a Schauder basis for $\mathcal{C}_{\text {Lip }}(S, 0)$.

For any $x \in S \backslash\{0\}$, let $m\left(f_{x}\right): S \times S \rightarrow \mathbb{R}$ be defined as follows:

$$
m\left(f_{x}\right)(y, z)=\left|f_{x}(y)-f_{x}(z)\right| .
$$

Then, clearly $m\left(f_{x}\right) \in \mathcal{P} \mathcal{M}_{\text {Lip }}(S)$ and the assignment

$$
f_{x} \mapsto m\left(f_{x}\right), x \in S \backslash\{0\},
$$

extends to a linear operator $m: \mathcal{C}_{\text {Lip }}^{+}(S, 0) \rightarrow \mathcal{P} \mathcal{M}_{\text {Lip }}(S)$ by the formula

$$
m\left(\sum_{x \in S \backslash\{0\}} \alpha_{x} f_{x}\right)=\sum_{x \in S \backslash\{0\}} \alpha_{x} m\left(f_{x}\right) .
$$

If $h \in \mathcal{C}_{\text {Lip }}^{+}(S, 0)$, define $v(h)(y)=u(m(h))(y, 0)$, for any $y \in X$. If $h \in \mathcal{C}_{\text {Lip }}(S, 0)$, we represent $h$ as $h=h_{1}-h_{2}$, where $h_{1}, h_{2} \in \mathcal{C}_{\text {Lip }}^{+}(S, 0)$, and define $v(h)=v\left(h_{1}\right)-v\left(h_{2}\right)$. One can easily see that then $v(h)$ is well-defined.

If $h \in \mathcal{C}_{\text {Lip }}(S)$, then $h-h(0) \in \mathcal{C}_{\text {Lip }}(S, 0)$ and we put $v(h)=v(h-h(0))+h(0)$. By a direct verification we show that $v: \mathcal{C}_{\text {Lip }}(S) \rightarrow \mathcal{C}_{\text {Lip }}(X)$ is a linear extension operator with $\|v\|<\infty$. Therefore $\lambda(S, X)<\infty$. 
For every $n$, denote by $r_{n}: X \rightarrow X_{n}$ the retraction that sends the complement of $X_{n}$ to $0 \in X_{n}$. Then evidently, $r_{n}$ is a 1-Lipschitz retraction, $r_{n}(S)=S_{n}$ and, by Lemma 3.2 , $\lambda\left(S_{n}, X_{n}\right) \leq \lambda(S, X)$. This obviously contradicts the inequality (4.1).

It follows by Proposition 3.3 that for the subset $S$ of the space $X$ from the proof of Theorem 4.1 there is no continuous linear operator extending Lipschitz metrics.

\section{Epilogue}

One can follow the proof of Theorem 4.1 and conjecture that $\lambda(S, X)<\infty$, whenever $\Lambda(S, X)<\infty$, for any subset $S$ of a metric space $X$. Actually, the following question arises:

Question 5.1. Are the numbers $\Lambda(S, X)$ and $\lambda(S, X)$ always equal?

We conjecture that the answer is affirmative. However, if this is not the case, then one can ask for a pseudometric analogue of any result concerning linear extensions of Lipschitz functions. As an example, we formulate the following question inspired by results from [9].

Question 5.2. Let $(X, d)$ be a metric space and $\omega: \mathbb{R}_{+} \rightarrow \mathbb{R}_{+}$be a concave non-decreasing function with $\omega(0)=0$. The function $d_{\omega}=\omega \circ d$ is a metric on $X$. Are the properties $\Lambda\left(X, d_{\omega}\right)<\infty$ and $\Lambda(X, d)<\infty$ equivalent?

It was proved in [15] that there exists a linear operator which extends partial pseudometrics with variable domain. The following question is, in some sense, a strengthening of Question 5.1. Given a compact metric space $X$, we let

$$
\mathcal{P} \mathcal{M}_{\text {Lip }}=\bigcup\left\{\mathcal{P} \mathcal{M}_{\text {Lip }}(A) \mid A \text { is a nonempty closed subset of } X\right\} .
$$

One can endow $\mathcal{P} \mathcal{M}_{\text {Lip }}$ with the following metric, $D$ :

$$
D\left(\varrho_{1}, \varrho_{2}\right)=\inf \left\{\left\|\tilde{\varrho}_{1}-\tilde{\varrho}_{2}\right\|_{X} \mid \tilde{\varrho}_{i} \text { is a Lipschitz extension of } \varrho_{i}\right\} \text {. }
$$

Question 5.3. Suppose that for a metric space $X, \Lambda(X)<\infty$. Is there a continuous linear extension operator for partial Lipschitz pseudometrics on $X$, i.e. a map $u: \mathcal{P} \mathcal{M}_{\text {Lip }} \rightarrow \mathcal{P} \mathcal{M}_{\text {Lip }}(X)$ which is continuous with respect to the metric $D$ and whose restriction onto every $\mathcal{P} \mathcal{M}_{\text {Lip }}(A)$, where $A$ is a subset of $X$, is linear?

Note that the question which corresponds to the one above for the case of partial Lipschitz fuctions is also open - see [10] for the results on simultaneous extensions of partial continuous functions.

Question 5.4. The space $\mathcal{P} \mathcal{M}_{\text {Lip }}(X)$ can also be endowed with the topologies of the uniform and pointwise convergence. Are there linear continuous extensions operators from $\mathcal{P} \mathcal{M}_{\text {Lip }}(A)$ to $\mathcal{P} \mathcal{M}_{\text {Lip }}(X)$, where $A$ is a subset of $X$, which are continuous in these topologies?

We denote the set of all Lipschitz ultrametrics on a subset $Y$ of a zero-dimensional metric space by $\mathcal{U} \mathcal{M}_{\text {Lip }}(Y)$. It was proved in [16] (see also [14]) that there exists a continuous extension operator that extends ultrametrics defined on a closed subspace of a zero-dimensional compact metric space and preserves the operation max.

Question 5.5. Given a subset $A$ of a zero-dimensional metric space $X$, is there a continuous extension operator for Lipschitz ultrapseudometrics $u: \mathcal{U} \mathcal{M}_{\text {Lip }}(A) \rightarrow \mathcal{U} \mathcal{M}_{\text {Lip }}(X)$ which preserves the operation max (is homogeneous)?

\section{REFERENCES}

[1] R.A. Alo, H.L. Schapiro, Extensions of totally bounded pseudometrics, Proc. Amer. Math. Soc. 19:4 (1968), $877-884$.

[2] R. Arens, Extension of coverings, of pseudometrics, and of linear-space-valued mappings, Canadian J. Math. 5 (1953), 211-215.

[3] T. Banakh, AE(0)-spaces and regular operators extending (averaging) pseudometrics, Bull. Polish Acad. Sci. 42 (1994), 107-206. 
[4] T. Banakh, N. Brodskiy, I. Stasyuk, E. D. Tymchatyn, On continuous extension of uniformly continuous functions and metrics, Colloq. Math. 116 (2009), 191-202.

[5] C. Bessaga, Functional analytic aspects of geometry. Linear extending of metrics and related problems, Progress in Functional Analysis (Peñíscola, 1990), pp. 247-257, North-Holland Math. Stud., 170, NorthHolland, Amsterdam, 1992.

[6] C. Bessaga, On linear operators and functors extending pseudometrics. Fund. Math. 142:2 (1993), 101-122.

[7] A. Brudnyi, Yu. Brudnyi, Metric spaces with linear extensions preserving Lipschitz condition, American Journal of Mathematics, Volume 129, Number 1, 2007, 217-314.

[8] Yu. Brudnyi, P. Shvartsman, The Whitney problem of existence of a linear extension operator, J. Geom. Anal. 7:4 (1997), 515-574.

[9] Yu. Brudnyi, P. Shvartsman, Stability of the Lipschitz extension property under metric transforms, Geom. Funct. Anal. 12:1, (2002), 73-79.

[10] H.-P. Künzi, L. B. Shapiro, On simultaneous extension of continuous partial functions, Proc. Amer. Math. Soc. 125 (1997), 1853-1859.

[11] J. Luukkainen, Extension of spaces, maps, and metrics in Lipschitz topology, Ann. Acad. Sci. Fenn. Ser. A I Math. Dissertationes No. 17 (1978), 62 pp.

[12] H. L. Schapiro, Extensions of pseudometrics, Canad. J. Math. 18 (1966), 981-998.

[13] L. I. Sennott, Extending complete continuous pseudometrics, Colloq. Math. 41 (1979), 237-241.

[14] I. Z. Stasyuk, On a homogeneous operator extending partial ultrametrics, Matematychni Studii, 22 (2004) $73-78$.

[15] E. D. Tymchatyn, M. Zarichnyi, On simultaneous linear extensions of partial (pseudo)metrics, Proc. Amer. Math. Soc. 132 (2004), 2799-2807.

[16] E. D. Tymchatyn, M. Zarichnyi, A note on operators extending partial ultrametrics, submitted.

[17] M. Zarichnyi, Regular linear operators extending metrics: a short proof, Bull. Pol. Acad. Sci. 44 (1996), $267-269$.

Faculty of Mathematics and Physics, and Faculty of Education, University of Ljubljana, P.O.B. 2964, 1001 Ljubljana, Slovenia

E-mail address: dusan.repovs@guest.arnes.si

Department of Mechanics and Mathematics, Lviv National University, Universytetska Str. 1, 79000 Lviv, Ukraine, and, Institute of Mathematics, University of Rzeszów, Rejtana 16 A, 35-310 Rzeszów, POLAND

E-mail address: mzar@litech.lviv.ua 